\title{
THE SHARPENING OF A RESULT CONCERNING PRIMITIVE IDEALS OF AN ASSOCIATIVE RING
}

\author{
F. SZÁSZ
}

The importance of the concept of primitive ideals of associative rings consists in the well-known theorem stating that every semisimple ring $A$ is a subdirect sum of primitive rings $B_{\nu}$, where a ring $A$ is called semisimple (in the sense of Jacobson) if the Jacobson radical, i.e. the intersection of all primitive ideals, coincides with the zero ideal $(0)$, and a ring $B_{\nu}$ is called primitive if the ideal $(0)$ is a primitive ideal of $B_{\nu}$. (Cf. N. Jacobson, Structure of rings, Colloq. Publ., Vol. 37, Amer. Math. Soc., Providence, R. I., 1956.)

Some new characterizations were recently given for the Jacobson radical of a ring $A$. For instance, A. Kertész [3] has shown in these Proceedings (generalizing an observation of L. Fuchs [1]) that the Jacobson radical $J$ of a ring $A$ consists of exactly those elements $x$ of $A$ for which the product $y x$ lies with every $y \in A$ in the Frattini $A$ submodule of the ring $A$, as of an $A$-right module $A$ for itself (cf. also Hille [2]). Furthermore A. Kertész [4] has shown that $J$ is the intersection of all those maximal right ideals $R$ of $A$ for which there must exist, for any element $x \in R(x \in A)$, a second element $y \in A$ with $y x \in R$; that is, those right ideals for which $A^{-1} R \subseteq R$ holds, where $X^{-1} R=\{y ; y \in A, X y \subseteq R\}$ for an arbitrary subset $X$ of $A$. Furthermore, let $L \cdot Y^{-1}$ denote the subset $\{z ; z \in A, z Y \subseteq L\}$.

Every modular right ideal $R$ of $A$ is quasi-modular in the sense that $A^{-1} R \subseteq R$ holds. The concept of quasi-modularity of right ideals $R$ was introduced in [6]. Solving a problem proposed by Kertész [4] I have shown in [6] the existence of an associative ring which has a quasi-modular maximal but not a modular right ideal. In my other paper [7] a two-sided ideal $Q$ of $A$ is called quasi-primitive if there exists a quasi-modular maximal right ideal $R$ of $A$ with $Q=A^{-1} R \subseteq R$. Obviously every primitive ideal is also quasi-modular in $A$, and almost trivially every artin ring with (0) quasi-primitive ideal is a total matrix ring over a skew field. Furthermore, any quasi-primitive ideal is clearly a prime ideal, and any commutative ring with (0) quasi-primitive ideal is a field.

Solving a problem of my colleague Dr. Steinfeld, I have proved in [7] that the Jacobson radical $J$ of $A$ must coincide with the intersec-

Received by the editors July 20, 1966. 
tion of all quasi-primitive ideals. There are two proofs of this fact in [7], an entirely elementary proof without quasi-regular element and irreducible modules, and (in a footnote) a second short proof with quasi-regular elements too.

In my note [7] some open problems on quasi-primitive ideals are mentioned, which have recently been solved completely by Dr. Steinfeld. He has shown that the concepts of primitivity and quasi-primitivity of ideals of arbitrary associative rings must coincide. Using a lemma which is proved but not explicitly announced in [7], Dr. Steinfeld has proved that there exists for every fixed quasi-modular maximal right ideal $R$ of $A$ an element $X$ of $A$ for which the right ideal quotient $R_{x}=\{x\}^{-1} R$ is a modular maximal right ideal of $A$ such that $A^{-1} R=A^{-1} R_{x}=(x A)^{-1} R$, which means that every quasi-primitive ideal $Q=A^{-1} R$ is by $Q=A^{-1} R_{x}$ also primitive in $A$.

This result of Dr. Steinfeld can be sharpened as follows:

ThEOREM. If $R$ is a quasi-modular maximal right ideal of an arbitrary associative ring, and if $x \in A$ is an arbitrary element of $A$ with the condition $x \notin R$, then the quasi-primitive ideal $Q=A^{-1} R$ coincides with the primitive ideal $P_{x}=A^{-1} R_{x}=(x A)^{-1} R$ of $A$ (instead of a single $x$ for any $x \in R)$.

Proof. In my note [7] it is shown that $R_{x}=\{x\}^{-1} R$ is a modular maximal right ideal of $A$ for every quasi-modular maximal right ideal $R$ of $A$ and for every $x \in A$ with $x \in R$. Namely, $R_{x}=\{x\}^{-1} R$ is a right ideal of $A$. By the quasi-modularity of $R, A^{2}+R=A$, and therefore we obtain $R A^{-1}=R$; that is, $x A+R=A$ for any $x \notin R$, $x \in A$. Since there exists for $x \notin R$ an element $y \in A$ with $x y \notin R$, the right ideal $R_{x}$ has the property $y \notin R_{x}$, i.e. $R_{x} \neq A$. If $z \in A$ is any element with $z \notin R_{x}$, one has by $x z \notin R$ obviously $x z A+R=A$, and thus for any $b \in A$ the existence of $a \in A$ and $r \in R$ with $x z a+r=x b$, and thereby also $x(b-z a)=r \in R, \quad b-z a \in R_{x}, \quad b \in z A+R_{x}$ and $A=z A+R_{x}$, which means the maximality of $R_{x}$ in $A$. Moreover, one has $x z a_{1}+r_{1}=x$ with some $a_{1} \in A$ and $r_{1} \in R$, which implies $x\left(1-z a_{1}\right) A$ $\subseteq R$, consequently $\left(1-z a_{1}\right) A \subseteq R_{x}$ and the modularity of the maximal right ideal $R_{x}$ of $A$.

By $x A+R=A$ and $A\left((x A)^{-1} R\right)=(x A+R)\left((x A)^{-1} R\right) \subseteq R$ one has on one side $(x A)^{-1} R \subseteq A^{-1} R$. On the other hand the condition $y \in A^{-1} R$ implies by $A^{-1} R=(x A+R)^{-1} R$ obviously $x A y \subseteq R$, that is $y \in(x A)^{-1} R$, and thus holds $A^{-1} R=(x A)^{-1} R$ for every $x \in R(x \in A)$. But one has almost trivially $(x A)^{-1} R=A^{-1}\{x\}^{-1} R=A^{-1} R_{x}$ too, which means that $Q=A^{-1} R=(x A)^{-1} R$ and $P_{x}=A^{-1} R_{x}$ must be for every $x \notin R$ the same primitive ideals of $A$. Q.E.D. 


\section{REFERENCES}

1. L. Fuchs, A remark on the Jacobson radical, Acta Sci. Math. Szeged 14 (1952), 167-168.

2. E. Hille, Functional analysis and semi-groups, Colloq. Publ., Vol. 31, Amer. Math. Soc., Providence, R. I., 1948; esp. p. 486 and Theorem 22.15.3.

3. A. Kertész, A characterization of the Jacobson radical, Proc. Amer. Math. Soc. 14 (1963), 595-597.

4. —, Über Artinsche Ringe, Akadémiai Kiad6, Budapest, 1966.

5. O. Steinfeld, Eine Bemerkung über die primitiven Ideale eines Ringes, Acta Math. Acad. Sci. Hungar. (to appear).

6. F. Szász, Die Lösung eines Problemes bezüglich einer Charakterisierung des Jacobsonschen Radikales, Acta Math. Acad. Sci. Hungar. (to appear).

7. - Eine Charakterisierung des Jacobsonschen Radikales eines Ringes, Bull. Acad. Polon. Sci. 152 (1967), 53-56.

8. - Radikalbegriffe für Halbgruppen mit Nullelement, die dem Jacobsonschen ringtheoretischen Radikal ähnlich sind, Math. Nachr. (to appear). 